\title{
Análisis de los principales determinantes de la aglomeración industrial: el caso de la IED japonesa automotriz en México
}

\section{Analysis of the Main Determinants of Industrial Agglomeration: The Case of Japanese Automotive FDI in Mexico}

DOI: $10.32870 /$ mycp.v9i27.682

\section{Resumen}

En la presente investigación se analiza el comportamiento de aglomeración de la IED japonesa en el sector automotriz dentro del Bajío mexicano. Se utiliza una base de datos con información no publicada proveniente de la Secretaría de Economía, dicha información se obtuvo mediante una petición directa a la dependencia. A partir de esta base de datos se realiza un análisis utilizando econometría espacial que permite comprobar la existencia de dependencia espacial en los datos. De acuerdo con los resultados obtenidos, las variables de PIB per cápita, la disponibilidad laboral, la infraestructura en transportación y la distancia geográfica explican la aglomeración de la IED japonesa en el Bajío mexicano. La importancia de los resultados encontrados ayuda a entender las similitudes económicas que entre entidades ayudan a atraer inversión similar en estados vecinos. Además, se encontró presencia de efectos espaciales positivos de la IED japonesa. Esto señala que aumentos de inversión japonesa en un estado van de la mano con incrementos de inversión japonesa en estados vecinos, lo que podría ser evidencia de cooperación horizontal, aprovechamiento de economías de escala, desarrollo de mercados laborales y de proveedores especializados en la región del Bajío.

Palabras clave: IED japonesa, determinantes de aglomeración industrial, análisis espacial, sector automotriz mexicano.

\author{
Cinthia Dinorah Manzano-Ramos ${ }^{1}$ \\ Leo Guzmán-Anaya ${ }^{2}$
}

\begin{abstract}
The current research analyses the agglomeration behavior of Japanese Foreign Direct Investment in Mexico's automotive sector in the Bajio region. The study employs unpublished data obtained from Mexico's Secretariat of Economy. The database is used to carry out the empirical analysis using spatial econometric techniques and test the presence of spatial dependence in the variables of the model. According to the results, Gross Domestic Product (GDP) per capita, labor force, transportation infrastructure, and geographical distance explain Japanese FDI agglomeration in Mexico's Bajio region. The results also elucidate that economic similarities within entities may help attract investment in similar proportions to neighboring states. Additionally, positive spatial effects from Japanese FDI were found, suggesting that an increase in Japanese FDI in one state is followed by increments of Japanese investment in neighboring states. This may be evidence of horizontal cooperation and a result of Japanese firms taking advantage of scale economies and the development of specialized labor markets and suppliers in the Bajio region.
\end{abstract}

Keywords: Japanese FDI, industrial agglomeration determinants, spatial analysis, Mexican automotive

Artículo recibido el 29 de enero de 2020 y dictaminado el 10 de junio de 2020.

1. Universidad de Guadalajara, Centro Universitario de Ciencias Económico Administrativas (CUCEA). Periférico Norte 799 C.P. 45100, Zapopan, Jalisco, México. ORCID: https://orcid.org/0000-0001-54967805 Correo electrónico: cinthia011493@gmail.com

2. Universidad de Guadalajara, Centro Universitario de Ciencias Económico Administrativas (CUCEA). Periférico Norte 799 C.P. 45100, Zapopan, Jalisco, México. ORCID: https://orcid.org/0000-0002-56823175 Correo electrónico: leo.guzman8@gmail.com 


\section{Introducción}

La industria manufacturera en México ha presentado un crecimiento acelerado en los últimos años, principalmente en el sector automotriz. Lo anterior debido a la participación de Inversión Extranjera Directa (IED) en la industria, donde uno de los principales protagonistas inversores ha sido su socio asiático, Japón. En el año 2016 el país asiático se mantuvo como uno de los principales inversores de México, aportando más del $5 \%$ del total de los flujos de IED hacia México, ocupando la sexta posición en cuanto a los principales inversores extranjeros (Secretaría de Economía, 2017).

Respecto a la IED de origen japonés, el sector manufacturero concentra la mayor parte de los flujos de inversión y la industria automotriz particularmente representa la mayor concentración de la inversión. En la literatura científica, el análisis de aglomeración de la IED automotriz generalmente se enfoca en países desarrollados, especialmente Estados Unidos. Para el caso de estudios previos sobre IED japonesa, la literatura ha enfocado sus esfuerzos en el caso de China. En este sentido, estudios sobre la aglomeración de la IED en México son reducidos, especialmente cuando se toma el caso de IED proveniente de Japón.

Dentro de los análisis realizados previamente sobre aglomeración de IED japonesa, se puede señalar el estudio de Smith y Florida (1994), quienes encontraron que la aglomeración japonesa del sector automotriz se explica por la densidad manufacturera, los costos, impuestos y urbanización. La urbanización en particular otorga resultados que sugieren que empresas japonesas buscan lugares rurales para establecer nuevas empresas, respondiendo a menores costos y mejor calidad de vida. Zhou, Delios y Yang (2002), por otro lado, reportan que el desarrollo regional y la infraestructura de transporte son los principales determinantes de aglomeración.

Estudios empíricos previos han observado la preferencia por la aglomeración de la inversión proveniente de Japón, bajo diferentes circunstancias y en diferentes contextos. La gran mayoría de estos estudios encuentran un comportamiento de aglomeración de la IED japonesa y señalan que para empresas multinacionales niponas resulta importante aprovecharse de la aglomeración industrial en los países donde ubican filiales. Los beneficios no son sólo directos, sino también indirectos al tener establecimientos que les ofrecerán servicios en el país receptor. 
En cuanto al análisis de los determinantes de la aglomeración de la industria manufacturera, se ha encontrado que estudios previos se enfocan principalmente en variables como el Producto Interno Bruto (PIB) per cápita (Belderbos \& Carree, 2002; Kang \& Jiang, 2012; Head, Ries, \& Swenson, 1999); el fenómeno de Keiretsu (Belderbos \& Carree, 2002; Horn \& Cross, 2016; Head, Ries, \& Swenson, 1995; Head et al., 1999); el desarrollo de la infraestructura de transportación (Tokunaga \& Jin, 2007; K. Head et al., 1995; Smith \& Florida, 1994; Lien \& Filatotchev, 2015; Boudier-Bensebaa, 2005; Du, Lu, \& Tao, 2008; Zhou et al., 2002; Cheng \& Kwan, 2000); la distancia geográfica (Blanc-Brude, Cookson, Piesse, \& Strange, 2014; Blonigen, Davies, Waddell, \& Naughton, 2004); los costos laborales medidos por el salario (Belderbos \& Carree, 2002; Friedman, Gerlowski, \& Silberman, 1992; Guimarães, Figueiredo, \& Woodward, 2000) y la disponibilidad laboral (Alfaro \& Chen, 2014; Bobonis \& Shatz, 2007; Boudier-Bensebaa, 2005; Friedman et al., 1992; Guimarães et al., 2000; He, 2003; Head et al., 1999; Head et al., 1995; Hilber, 2007; Horn \& Cross, 2016; Kang \& Jiang, 2012; Kotabe, 1993; Smith \& Florida, 1994).

Por otra parte, para poder definir y plantear concretamente el fenómeno de aglomeración se debe partir de los planteamientos iniciales, como lo es el caso de Weber y Friedrich (1929), quienes propusieron la teoría de la localización de las industrias, la cual se enfoca en los factores regionales de localización que inciden sobre la determinación de localización de las industrias, lo que lleva a la aglomeración. Es necesario también hacer mención de los planteamientos de Marshall (1961), quien señala las ventajas sobre la división del trabajo mediante la concentración de un gran número de empresas en lo que denominó "distritos industriales".

Más adelante, Krugman, tomando las ideas de Marshall y Weber desarrolla la teoría de la nueva geografía económica, que de acuerdo con Krugman ésta se define como "la ubicación de los factores de producción en espacio" (Krugman, 1991, p. 483).

Tomando en cuenta lo señalado en la literatura, en el presente estudio se consideran los costos laborales, la disponibilidad laboral, el desarrollo de infraestructura, el PIB regional y la distancia geográfica, como variables que determinan la aglomeración de la IED automotriz japonesa.

Otros planteamientos teóricos y empíricos señalan también la importancia de aspectos contextuales como legislación vigente o clima político, social y cultural en la ubicación de empresas extranjeras. Estudios como los 
de Buchanan, Le y Rishi (2012) señalan que la calidad institucional está relacionada con niveles de IED. Sin embargo, empíricamente resulta complicado encontrar datos confiables para corroborar la influencia de políticas públicas en la atracción de IED, ya que generalmente estos datos no están disponibles (Jordaan, 2009; Ortega, 2015).

Para el caso de México, Ortega (2015) reporta en su estudio factores económicos que inciden en la atracción de IED, mientras que factores políticos, sociales y administrativos no tienen efectos significativos. Similarmente, el análisis de Tinoco-García y Guzmán-Anaya (2020) considera los factores regionales de atracción de IED en México y en el estudio se incluye la variable de políticas públicas regionales, medida por la calidad institucional estatal y niveles de corrupción. En los resultados del análisis, la variable no resulta estadísticamente significativa. Los autores señalan que la falta de resultados significativos se atribuye a que estas políticas generalmente cuentan con bajos presupuestos o sus metas no van de acuerdo con los recursos o esfuerzos destinados. Además, para el caso de México se resalta que existe una dificultad para disponer de datos, ya que los incentivos gubernamentales son negociados a puertas cerradas con poco o nulo registro de las negociaciones entre multinacionales y gobiernos en el país receptor. Canales (2010) señala además que políticas gubernamentales de atracción, inserción y retención de IED cuentan con poco tiempo de implementación ya que los gobiernos impulsan actividades con resultados a corto plazo. Lugo-Sánchez (2018) analiza el rol de las políticas públicas fiscales en atraer IED japonesa automotriz en los estados del Bajío mexicano. El estudio realizado mediante visitas a empresas japonesas encuentra que las políticas fiscales cumplen un rol importante pero no determinante en la ubicación de subsidiarias en México. Factores como demanda regional, costos regionales de producción y la presencia de economías de aglomeración son más importantes en la atracción de IED japonesa automotriz en el Bajío.

Es importante mencionar que para el caso específico de este estudio se estará planteando la medición de la aglomeración en función del histórico de flujos de IED de origen japonés en el sector automotriz, el número de empresas de origen japonés pertenecientes al sector automotriz y un promedio de estos dos, todo esto por entidad federativa en la zona del Bajío. Esta zona se delimitó a los estados de Aguascalientes, Guanajuato, Jalisco, Nuevo León, Querétaro, San Luis Potosí y Zacatecas, esto por la importancia relativa en términos de atracción de IED japonesa en el sector automotriz (ProMéxico, 2017). 
México se posiciona entre varios países como una opción viable y atractiva para el establecimiento de plantas manufactureras japonesas, sobre todo en el sector automotriz, propiciando la aglomeración en ciertos estados del país. Es por esto que el objetivo principal de esta investigación recae en analizar los principales determinantes de aglomeración identificando los efectos de cada variable sobre la decisión de localización de empresas japonesas. Conocer los determinantes de la formación de clústeres industriales tiene relevancia en política pública, ya que los gobiernos buscan fomentar la aglomeración industrial automotriz para poder generar oportunidades de integrar y mejorar las cadenas de suministro de la industria (ProMéxico, 2016).

El estudio se inscribe en la literatura relacionada con factores de localización de IED en países receptores. La literatura previa es aún limitada en explicar los patrones de localización de empresas multinacionales japonesas fuera de Asia (principalmente China) y Norteamérica (especialmente Estados Unidos), por lo que la investigación proporciona un análisis novedoso sobre la localización de IED japonesa en el caso de México. Contextualmente, el caso se vuelve relevante ya que la presencia de IED automotriz japonesa en el país ha aumentado considerablemente desde la entrada en vigor del Acuerdo de Asociación Económica México-Japón (AAE) en el año 2005 y este crecimiento se ha acelerado desde 2015. Lo anterior genera condiciones para un aporte en el ámbito académico y además se espera que los resultados puedan guiar la política pública relacionada con la atracción de IED en la región del Bajío mexicano.

Para finalizar, la presente investigación se delimita en la zona del Bajío, la cual ha sido la principal receptora de IED automotriz japonesa, especialmente con la llegada de empresas ensambladoras y proveedoras Tier-1 en la región. Los hallazgos de esta investigación buscarán comprobar dependencia espacial en este tipo de inversión, al comprobar que las características económicas y regionales explican la aglomeración de IED japonesa en la industria automotriz.

\section{Revisión de literatura}

En general la literatura señala que la decisión de la IED sobre establecerse en cierta región está basada en gran medida en la aglomeración existente. Según Porter (1986), las multinacionales tienden a colocarse cerca unas de otras aprovechando las ventajas competitivas que surgen por la presencia de clústeres. 
El fenómeno de aglomeración o también llamado clúster, es un factor que representa la manera en la cual se localizan empresas de la misma procedencia o que pertenecen al mismo sector. Dentro de la revisión que se ha hecho en la literatura empírica es posible encontrar que el análisis de clústeres se ha abordado de diferentes formas, dando pie a distintas concepciones, dependiendo del estudio y del autor.

Aunque el presente texto no se enfoca en el análisis de clústeres desde el punto de vista de cadenas de valor en el sector manufacturero, es importante mencionarlo, ya que es un aspecto fundamental en términos de productividad para las empresas y también proporciona un punto importante que recae sobre cada una de las variables utilizadas para el estudio. Tal como se mencionó con anterioridad, las multinacionales tienden a colocarse cerca, aprovechando ventajas competitivas (Porter, 1986), lo que permite la creación de los clústeres.

De manera más específica dentro de la industria automotriz, los clústeres generan economías de escala que contribuyen a la disminución de costos, la colaboración y la transferencia tecnológica, así como a la vinculación entre empresas a nivel nacional e internacional (ProMéxico, 2017). Aquí es donde recae la importancia de los clústeres, ya que permiten la atracción de inversión extranjera al desarrollarse las características mencionadas, provocando una acción recíproca en el sector, al permitir la transferencia tecnológica generando avances en lo que respecta a los centros de investigación y desarrollo dentro de dichos clústeres.

En la literatura empírica que analiza a la IED proveniente de Japón, resalta el estudio hecho por Belderbos y Carree (2002), pionero en proponer distintas conceptualizaciones de la aglomeración de las plantas manufactureras. Específicamente, en su estudio se plantean tres tipos de aglomeración: el primero es a nivel industria, el segundo por la nacionalidad y el último por el fenómeno del Keiretsu. ${ }^{3}$

Los hallazgos reportados por Belderbos y Carree (2002) señalan que la probabilidad de que una empresa elija una región es afectada de manera positiva por la presencia de establecimientos del mismo tipo. Asimismo, los autores también argumentan que el impacto de aglomeración es mayor al considerar

3. El empleo de la palabra Keiretsu se refiere a clústeres independientes de empresas que desarrollan actividades similares, regularmente actividades manufactureras. Hay dos tipos principales de Keiretsu: el "horizontal", que es también llamado financiero, y el "vertical" (Lincoln y Shimotani, 2010). 
las variables regionales siguientes: tamaño económico regional, potencial de demanda, nivel salarial, infraestructura, distancia de Japón a empresa filial e incentivos a la inversión. En este último punto es importante señalar que la literatura empírica generalmente no incluye los incentivos a la inversión en sus análisis, principalmente por la dificultad de obtener datos confiables sobre esta variable, ya que gran parte de estas negociaciones entre el sector gubernamental y las multinacionales se realiza a puertas cerradas.

Las características regionales ejercen un papel importante en estudios sobre aglomeración de IED. El tamaño económico regional medido por el PIB es utilizado de manera recurrente en diferentes estudios. De acuerdo con Belderbos y Carree (2002), la importancia de considerar el PIB surge en poder asegurar que los coeficientes de las variables de aglomeración no estén sesgados hacia arriba, ya que los conteos de aglomeración están correlacionados con el tamaño de la región.

Las condiciones del mercado laboral están estrechamente relacionadas con la decisión de aglomeración de IED, considerando que las empresas japonesas plantean establecerse en lugares con disponibilidad de mano de obra capacitada. En este sentido, estudios previos sugieren que empresas japonesas tienden a colocarse en lugares con salarios altos, reflejando una compensación en favor de un capital humano más capacitado y una mayor estabilidad laboral (Smith \& Florida, 1994).

La variable de infraestructura de transporte adquiere gran importancia dentro de la cultura japonesa de producción "just in time", es por esto que la IED se ubica en lugares con una infraestructura para el transporte desarrollada que les permita trasladar sus productos, facilitando la comunicación entre los usuarios y proveedores dentro de la cadena productiva (Smith \& Florida, 1994). Es importante mencionar que Smith y Florida (1994) plantean dentro de su análisis que la variable de infraestructura de transporte tiene un peso significativo más alto cuando la industria es de materiales pesados; por ejemplo, en la industria automotriz. Además, Zhou et al. (2002) enfatizan la importancia de la infraestructura de transporte como una variable determinante en la decisión de establecer plantas japonesas en un país al representar un factor necesario para acceder al mercado, y facilitar las exportaciones. Similarmente, Boudier-Bensebaa (2005) encontró que la infraestructura de transporte es una variable de significancia positiva para la aglomeración de las plantas extranjeras, mencionando que esta variable facilita la comunica- 
ción y trae beneficios para la entrada a nuevos mercados y la difusión de la información.

En estudios sobre aglomeración de IED es necesario incluir la dimensión espacial, específicamente la distancia geográfica dentro del análisis, como variable explicativa de este fenómeno. De acuerdo con Blanc-Brude et al. (2014), es necesario incluir la variable de distancia geográfica para poder incluir los pesos de matrices dentro del análisis de regresión espacial, al igual que comprobar la dependencia espacial existente en las variables que son objeto de estudio. Por otro lado, Blonigen et al. (2004) indican que al utilizar un modelo donde se esté considerando a varios estados y la influencia de unos sobre otros, se debe realizar un análisis de dependencia espacial, considerando la distancia geográfica.

\section{Metodología empírica}

Tomando en cuenta lo mencionado en la literatura, se optó por utilizar modelos de regresión espacial para controlar la posible correlación espacial en los datos. Los modelos estimados son el modelo de autorregresión espacial (SAR, por sus siglas en inglés), modelo de error espacial (SEM, por sus siglas en inglés) o con la inclusión de ambos modelos, donde se represente la autorregresión espacial (SAR) y se mantenga el término de error espacial (SEM); este modelo es llamado SAC (Spatially Autocorrelated Errors) (Belotti, Hughes \& Mortari, 2017). Estos modelos fueron elegidos debido a que la dependencia espacial que se plantea comprobar en este tipo de estudios se puede representar con una variable dependiente con rezago espacial (SAR), con un término de error de correlación espacial (SEM), o con la inclusión de ambos (Belotti, Hughes \& Mortari, 2017).

El parámetro $(\rho)$ obtenido a partir del modelo SAR muestra la fuerza del efecto de la IED de los estados vecinos sobre la IED del estado $i$; en cuanto al modelo SEM, éste muestra su parámetro como el término de error de correlación espacial $\lambda$.

El modelo SAR se describe de acuerdo con la siguiente ecuación general:

$$
Y=\rho W Y+X \beta+\epsilon
$$

Donde:

Y: representa la variable dependiente del modelo. 
$\mathrm{X}$ : es el conjunto de variables independientes.

$\mathrm{W}$ : representa la matriz de pesos espaciales, indicando la conectividad entre los estados.

$\epsilon$ : término de error.

Tomando en cuenta la ecuación 1, el modelo SAR para el presente análisis queda de la siguiente manera para siete estados del Bajío (Aguascalientes, Guanajuato, Jalisco, Nuevo León, Querétaro, San Luis Potosí y Zacatecas) (i) en el año $(t)$. Al reemplazar las variables de estudio:

$\operatorname{IED}_{i t}=\rho W_{i j}$ IED $_{t}+\beta 1$ pibpc $_{i t}+\beta 2$ salar $_{i t}+\beta 3$ dislab $_{i t}+\beta 4$ transno $_{i t}+\beta 5$ distgeo ${ }_{i t}+\epsilon$

La descripción de las variables y sus fuentes se presentan en la tabla 1.

En relación con el modelo SEM, éste se describe con la siguiente ecuación general:

$Y_{i t}=X_{i t} \beta+\mu_{i t}+V_{i t}$

Donde V se entiende como el término de error de autocorrelación espacial, que se plantea de la siguiente manera:

$V_{i t}=\lambda W V_{j t}+\epsilon_{i t}$

Dicho lo anterior, el modelo SEM se define en el presente estudio como:

IED $_{i t}=\beta$ pibpc $_{i t}+\beta 2$ salar $_{i t}+\beta 3$ dislab $_{i t}+\beta 4$ transno $_{i t}+\beta 5$ distgeo $_{i t}+V_{i t}$

En cuanto al modelo SAC, éste se representa con la siguiente ecuación:

$Y_{i t}=\rho \mathrm{Wy}_{\mathrm{jt}}+X_{i t} \beta+\mu_{i t}+V_{t}$

Para el modelo SAC se entiende que el símbolo $\mathrm{V}$ representa de igual forma el término de error de autocorrelación espacial, sólo que difiere del modelo SEM en la inclusión de $\rho$. 


\section{Tabla 1}

\section{Descripción de variables}

\begin{tabular}{|c|c|c|c|}
\hline \multicolumn{4}{|l|}{ Variable dependiente } \\
\hline Variable & \multicolumn{2}{|l|}{ Descripción } & Recursos \\
\hline \multirow[t]{3}{*}{$\begin{array}{l}\text { ied japonesa al sector } \\
\text { automotriz } \\
\text { (ied, noemp, iedemp) }\end{array}$} & \multicolumn{2}{|c|}{$\begin{array}{l}\text { Flujos de IED japonesa anual al sector } \\
\text { automotriz mexicano en los siete } \\
\text { estados del Bajío (1999-2017) } \\
\text { (ied) }\end{array}$} & \multirow[t]{3}{*}{$\begin{array}{l}\text { Información de la } \\
\text { Secretaría de Economía }{ }^{1} \\
(1999-2017)\end{array}$} \\
\hline & \multicolumn{2}{|c|}{$\begin{array}{l}\text { El número de empresas de IED } \\
\text { japonesa automotriz en los siete } \\
\text { estados del Bajío hasta el año } \\
\text { determinado de estudio (1999-2017) } \\
\text { (noemp) }\end{array}$} & \\
\hline & \multicolumn{2}{|c|}{$\begin{array}{l}\text { Promedio anual de IED japonesa al } \\
\text { sector automotriz mexicano por } \\
\text { empresa en los siete estados del Bajío } \\
\text { (1999-2017) } \\
\text { (iedemp) }\end{array}$} & \\
\hline \multicolumn{4}{|l|}{ Variables independientes } \\
\hline Variable & $\begin{array}{l}\text { Significancia } \\
\text { esperada }\end{array}$ & Descripción & Recursos \\
\hline $\begin{array}{l}\text { PIB per cápita } \\
\text { (pibpc) }\end{array}$ & $(+)$ & $\begin{array}{l}\text { Producto interno bruto } \\
\text { per cápita anual en los } \\
\text { siete estados del Bajío } \\
\text { (1999-2017) }\end{array}$ & $\begin{array}{l}\text { Contenido estadístico de } \\
\text { INEGI (1999-2017) }\end{array}$ \\
\hline $\begin{array}{l}\text { Costos Salariales } \\
\text { (salar) }\end{array}$ & $(+)$ & $\begin{array}{l}\text { Salarios mínimos } \\
\text { vigentes anuales en los } \\
\text { siete estados del Bajío } \\
(1999-2017)\end{array}$ & $\begin{array}{l}\text { Comisión Nacional } \\
\text { de Salarios Mínimos } \\
\text { Vigentes } \\
(1999-2017)\end{array}$ \\
\hline $\begin{array}{l}\text { Disponibilidad laboral } \\
\text { (dislab) }\end{array}$ & $(+)$ & $\begin{array}{l}\text { Promedio anual de } \\
\text { desocupación laboral en } \\
\text { los siete estados del Bajío } \\
(1999-2017)\end{array}$ & $\begin{array}{l}\text { Contenido estadístico de } \\
\text { INEGI (ENE y ENOE) } \\
(1999-2017)\end{array}$ \\
\hline $\begin{array}{l}\text { Infraestructura } \\
\text { en transportación } \\
\text { (transno) }\end{array}$ & $(+)$ & $\begin{array}{l}\text { Longitud de red federal } \\
\text { de autopistas anual en } \\
\text { los siete estados del } \\
\text { Bajío, entre su respectiva } \\
\text { área geográfica (1999- } \\
\text { 2017) }\end{array}$ & $\begin{array}{l}\text { Anuario estadístico } \\
\text { de la Secretaría de } \\
\text { Comunicaciones y } \\
\text { Transportes (1999-2017) }\end{array}$ \\
\hline
\end{tabular}


Análisis de los principales determinantes de la aglomeración industrial: el caso de la IED japonesa automotriz en México

\begin{tabular}{l|l|l|l}
\hline Variables independientes & $\begin{array}{l}\text { Significancia } \\
\text { esperada }\end{array}$ & Descripción & Recursos \\
\hline Variable & $(-)$ & $\begin{array}{l}\text { Distancia geográfica } \\
\text { de los siete estados } \\
\text { del Bajío desde su } \\
\text { Distancia geográfica } \\
\text { (distgeo) }\end{array}$ & $\begin{array}{l}\text { Herramienta de Google } \\
\text { Mapas } \\
\text { la frontera con Estados } \\
\text { Unidos (Nuevo Laredo, } \\
\text { Tamaulipas) }\end{array}$ \\
\hline
\end{tabular}

Fuente: elaboración propia.

${ }^{1}$ Se utiliza una base de datos con información no publicada proveniente de la Secretaría de Economía; dicha información se obtuvo mediante una petición directa a la dependencia.

\section{Resultados}

Para realizar la estimación de los modelos se convirtieron las cifras a logaritmos. Los resultados obtenidos muestran coeficientes significativos para la mayoría de las variables. Es importante mencionar que para determinar cuál de los modelos mejor se ajusta a los datos, se tomó en consideración el Akaike's Information Criterion (AIC).

Se compararon los resultados de los modelos SAR, SEM y SAC, y se determinó mediante el AIC el uso del modelo SAR. Sin embargo, se incluyen los resultados de los tres modelos, especialmente porque en la literatura empírica se sugiere y se da preferencia al modelo SEM en este tipo de estudios (BlancBrude et al., 2014; Coughlin \& Segev, 2000).

En cuanto a la selección del tipo de efecto a utilizar en el modelo SAR, éste presentó resultados significativos para las tres formas de medición de la variable dependiente. Para el caso del modelo con la variable dependiente "ied", se presentaron resultados con un peso AIC de más de $64 \%$ a favor del modelo SAR con efectos aleatorios. En lo que respecta al uso de la variable dependiente "número de empresas" se reportaron resultados a favor del modelo SAR con efectos aleatorios, con poco más del $50 \%$ de peso AIC. En cuanto al uso del "promedio anual de IED por número de empresas", los modelos señalan resultados a favor del modelo SAR con efectos aleatorios con $77 \%$ de peso AIC. De acuerdo con los resultados obtenidos, se estimaron los modelos SAR y SEM bajo efectos aleatorios. Para el caso del modelo SAC, la estimación se realizó con efectos fijos, de acuerdo con el peso AIC. 
De acuerdo con los resultados obtenidos en los modelos 1 , 2 y 3 correspondientes a la variable ied, que representa los flujos de IED japonesa al sector automotriz mexicano por estado; el valor de AIC indica que el modelo que mejor se ajusta es el SAR con efectos aleatorios (modelo 1). Sin embargo, se debe también reportar y analizar el modelo SEM, dada la significancia de los coeficientes en todas las variables y por lo señalado por Coughlin y Segev (2000) y Blanc-Brude et al. (2014), quienes especifican que el modelo SEM es el que mejor se adecua para este tipo de análisis. El modelo SAC también se incluye de referencia para compararse con los otros modelos.

Analizando los resultados de la tabla 2, en lo que respecta al valor del coeficiente autorregresión espacial ( $\rho$ ), éste presenta una significancia relevante para el modelo 1 del 95\%; de igual forma se presentan resultados significativos para el valor de error de correlación espacial $(\lambda)$ con significancia del 95 y $99 \%$ para los modelos 2 y 3 , respectivamente. De acuerdo con estos resultados, se puede afirmar la existencia de dependencia espacial en los datos. Estos hallazgos concuerdan con estudios previos sobre IED, especialmente cuando los estados tienen características económicas similares, como es el caso de la zona del Bajío (Blonigen et al., 2004; Coughlin \& Segev, 2000; Fukao, Kravtsova \& Nakajima, 2014; Horn \& Cross, 2016; Villaverde \& Maza, 2015).

En lo que respecta a los resultados del modelo 1, se presentaron coeficientes significativos para todas las variables de interés, con excepción de la variable que especifica los salarios. Los resultados se mantienen consistentes en el modelo 2 (SEM) con coeficientes significativos en las variables de interés, exceptuando la de los salarios. En el modelo 2 la significancia estadística mejora al 99\%, lo que podría indicar resultados más robustos, como lo indica la literatura previa.

Analizando los coeficientes individualmente, la variable correspondiente al PIB per cápita registra un valor de 3.26 en el modelo número 1 y un valor de 3.68 en el modelo 2. Los resultados muestran una relación positiva y significativa entre el tamaño del mercado y la aglomeración de la IED. Específicamente incrementos porcentuales de $1 \%$ en el PIB per cápita estatal, acompañado de incrementos de más del 3\% en flujos de IED a los estados. Estos resultados van de acuerdo con lo señalado por Blanc-Brude et al. (2014) y por Coughlin y Segev (2000) respecto a la relación de atracción del PIB per cápita sobre la inversión proveniente de Japón, donde empresas niponas favorecen establecerse en lugares con características económicas similares. También con lo señalado por Villaverde y Maza (2015), quienes muestran la 
presencia de dependencia espacial en las características del mercado como factor de localización de la IED en algún país.

De acuerdo con los hallazgos realizados por Blanc-Brude et al. (2014), se comprueba que existe autocorrelación espacial en estados con características económicas parecidas, es por ello la significancia encontrada en la variable de PIB per cápita. En el caso de dicha variable se capturan los efectos del capital humano, el nivel de desarrollo de infraestructura no sólo de las carreteras sino en general, adquiriendo así el tamaño de mercado. Los resultados encontrados en esta variable explican gran parte de la dependencia espacial y concuerdan con los hallazgos de Blanc-Brude et al.; la inversión se establece en lugares con características económicas similares (Blanc-Brude et al., 2014).

Respecto a la variable disponibilidad laboral, ésta presentó un valor de -2.55 para el modelo 1 y un valor de -2.61 para el modelo 2, ambos estadísticamente significativos. Sin embargo, los coeficientes tienen el signo contrario al esperado. Lo anterior podría ser resultado de la preferencia de empresas japonesas por establecerse en mercados laborales especializados; es decir la mano de obra calificada puede ser escasa y va de acuerdo con disposición por pagar salarios más altos. Además, como lo señalan Blanc-Brude et al. (2014), la variable PIB per cápita puede en ciertos casos capturar los efectos del capital humano y no permitir observar su efecto de manera individual.

En lo que respecta a la variable de infraestructura en transportación (transno), los coeficientes resultaron significativos para los modelos $1 \mathrm{y}$ 2 , con valores de 7.65 y 6.83 , respectivamente, lo cual indica una relación positiva entre la presencia de infraestructura y la aglomeración de la IED japonesa. Estos hallazgos van de acuerdo con lo encontrado en la literatura, como lo señalado por Smith y Florida (1994), al tratarse de una industria de materiales pesados dicha variable toma un peso significativo. Por otro lado, de acuerdo con Boudier-Bensebaa (2005), la infraestructura es una variable de gran significancia positiva, ya que forma parte de la decisión de los japoneses para establecerse en alguna región, la cual a su vez se relaciona con el nivel de desarrollo económico en la región, al aportar beneficios para la entrada a nuevos mercados y difusión de la información, facilitando la comunicación.

La variable distancia de la frontera (distgeo) exhibe también el signo esperado y es estadísticamente significativa al 99\% de confianza en ambos modelos. Estos resultados son consistentes con la idea de que la IED japonesa en México se aglomera en estados en las regiones centro y norte de México, es decir la cercanía a Estados Unidos también influye en la decisión de localización 
de las firmas niponas. Estos resultados concuerdan con hallazgos empíricos previos que sugieren que la IED japonesa elige su ubicación tomando en cuenta la distancia hacia su mercado meta (Blanc-Brude et al., 2014; Coughlin \& Segev, 2000; Villaverde \& Maza, 2015).

En el modelo 1 se puede también resaltar el coeficiente $\rho$ con un valor de 0.2302 y significativo al $95 \%$ de confianza. El coeficiente indica la presencia de efectos espaciales positivos de la IED japonesa en la región del Bajío. Específicamente, el aumento de IED japonesa en $1 \%$ en uno de los estados del Bajío va de la mano con aumentos del $0.23 \%$ en estados vecinos. Este resultado resulta interesante ya que no parece que en la región del Bajío existan efectos competitivos por la IED entre estados, o lo que se conoce en la literatura como "crowding out".

Tabla 2

Resultados de la regresión con dependencia espacial (lnied)

\begin{tabular}{l|c|c|c}
\hline & Modelo 1 & Modelo 2 & Modelo 3 \\
\hline Variable & SAR & SEM & SAC \\
\hline PIB per cápita (lnpibpc) & $3.2618^{* *}$ & $3.6809^{* * *}$ & -1.1590 \\
\hline Costos salariales (lnsalar) & $(2.37)$ & $(2.77)$ & $(-0.18)$ \\
\hline & 7.5197 & 10.8374 & 19.5496 \\
\hline $\begin{array}{l}\text { Disponibilidad laboral } \\
\text { (lndislab) }\end{array}$ & $(0.62)$ & $(0.73)$ & $(0.91)$ \\
\hline & $-2.5517^{* * *}$ & $-2.6178^{* * *}$ & -0.8701 \\
\hline $\begin{array}{l}\text { Infraestructura en } \\
\text { transportación (lntransno) }\end{array}$ & $(-8.75)$ & $(-12.20)$ & $(-0.38)$ \\
\hline & $7.6534^{* * *}$ & $6.8318^{* * *}$ & -2.6152 \\
\hline $\begin{array}{l}\text { Distancia geográfica } \\
\text { (lndistgeo) }\end{array}$ & $(2.91)$ & $(3.15)$ & $(-0.45)$ \\
\hline & $-8.1720^{* * *}$ & $-7.2074^{* * *}$ & - \\
\hline Constante & $(-3.51)$ & $(-3.82)$ & - \\
\hline$\rho$ & 31.5322 & 8.6740 & - \\
\hline & $(0.41)$ & $(0.11)$ & - \\
\hline$\lambda$ & $0.2303^{* *}$ & & -0.3656 \\
\hline$\varphi$ & $(1.99)$ & & $(-1.56)$ \\
\hline & & $0.2541^{* *}$ & $0.5289^{* * *}$ \\
\hline & & $(2.19)$ & $(5.65)$ \\
\hline & & $-19.5235^{* * *}$ & \\
\hline & & & \\
\hline & & & \\
\hline & & & \\
\hline & & & \\
\hline & & & \\
\hline & & & \\
\hline & & & \\
\hline & & & \\
\hline
\end{tabular}


Análisis de los principales determinantes de la aglomeración industrial: el caso de la IED japonesa automotriz en México

\begin{tabular}{l|c|c|c}
\hline & Modelo 1 & Modelo 2 & Modelo 3 \\
\hline Variable & SAR & SEM & SAC \\
\hline & & $(-4.44)$ & \\
\hline AIC & 885.2755 & 884.1061 & 873.2192 \\
\hline Delta AIC & 12.0563 & 10.8869 & 0.0000 \\
\hline Pesos Akaike & 0.6412 & 0.3573 & 0.0015 \\
\hline Número de observaciones & 133 & 133 & 133 \\
\hline Número de grupos & 7 & 7 & 7 \\
\hline
\end{tabular}

Notas: (1) Variable dependiente: ied (flujos de IED japonesa al sector automotriz mexicano, por entidad federativa 1999-2017). (2) Números entre paréntesis indican z (o t para el caso de efectos fijos) estadístico. (3) Todos los datos se encuentran en logaritmos.

* Nivel de significancia en $90 \%$

** Nivel de significancia en $95 \%$

*** Nivel de significancia en $99 \%$

Fuente: elaboración propia.

Se estimaron modelos adicionales cambiando la variable dependiente a total de empresas automotrices japonesas por estado y al promedio estatal anual de IED japonesa automotriz por empresa nipona. Los modelos se estiman para corroborar la robustez de las estimaciones presentadas anteriormente. Los resultados muestran que el modelo SAR es el que otorga los valores AIC más altos, pero cercano a las estimaciones SEM en ambos casos. Los resultados se muestran en las tablas 3 y 4 .

Para la estimación tomando en cuenta el total de empresas como variable dependiente, en las estimaciones SAR y SEM (modelos 4 y 5) se confirma la importancia de la infraestructura del transporte en la aglomeración de las empresas japonesas automotrices. En ambos casos los coeficientes son positivos y estadísticamente significativos al 99\% de confianza. Los coeficientes muestran una elasticidad por arriba de 1 , indicando que incrementos porcentuales de infraestructura van de la mano de incrementos porcentuales mayores en número de empresas en el Bajío. Además, se mantiene la significancia estadística y signo negativo para la distancia geográfica, confirmando la influencia de la cercanía a Estados Unidos como factor de decisión de localización de las firmas niponas (véase tabla 3). 


\section{Tabla 3}

Resultados de la regresión con dependencia espacial (lnnoemp)

\begin{tabular}{|c|c|c|c|}
\hline & Modelo 4 & Modelo 5 & Modelo 6 \\
\hline Variable & $S A R$ & SEM & $S A C$ \\
\hline \multirow[t]{2}{*}{ PIB per cápita (lnpibpc) } & -0.5463 & -0.5632 & 0.0091 \\
\hline & $(-0.97)$ & $(-0.92)$ & $(0.02)$ \\
\hline \multirow[t]{2}{*}{ Costos salariales (lnsalar) } & 2.3104 & 2.4236 & 1.3169 \\
\hline & $(1.23)$ & $(1.13)$ & $(0.59)$ \\
\hline \multirow[t]{2}{*}{ Disponibilidad laboral (lndislab) } & -0.0167 & -0.0118 & -0.3122 \\
\hline & $(-0.11)$ & $(-0.08)$ & $(-1.21)$ \\
\hline \multirow{2}{*}{$\begin{array}{l}\text { Infraestructura en transportación } \\
\text { (lntransno) }\end{array}$} & $1.5028^{* * *}$ & $1.4821^{* * *}$ & $2.2875^{* *}$ \\
\hline & $(3.56)$ & $(3.94)$ & $(2.03)$ \\
\hline \multirow[t]{2}{*}{ Distancia geográfica (lndistgeo) } & $-2.0696^{* * *}$ & $-2.0525^{* * *}$ & - \\
\hline & $(-3.51)$ & $(-3.77)$ & - \\
\hline \multirow[t]{2}{*}{ Constante } & 15.0954 & 14.6979 & - \\
\hline & $(1.37)$ & $(1.37)$ & - \\
\hline \multirow[t]{2}{*}{$\rho$} & 0.0305 & & $0.2800^{* *}$ \\
\hline & $(0.29)$ & & $(2.50)$ \\
\hline \multirow[t]{2}{*}{$\lambda$} & & 0.0339 & -0.3390 \\
\hline & & $(0.27)$ & $(-1.21)$ \\
\hline \multirow[t]{2}{*}{$\varphi$} & & $-3.5232^{* *}$ & \\
\hline & & $(-2.56)$ & \\
\hline AIC & 340.7275 & 340.7207 & 325.8411 \\
\hline Delta AIC & 14.8864 & 14.8796 & 0.0000 \\
\hline Akaike weights & 0.5007 & 0.4990 & 0.0003 \\
\hline Número de observaciones & 133 & 133 & 133 \\
\hline Número de grupos & 7 & 7 & 7 \\
\hline
\end{tabular}

Notas: (1) Variable dependiente: noemp (número de empresas japonesas en el sector automotriz mexicano, por entidad federativa 1999-2017). (2) Números en paréntesis indican z (o t para el caso de efectos fijos) estadístico. (3) Todos los datos se encuentran en logaritmos.

* Nivel de significancia en $90 \%$

** Nivel de significancia en $95 \%$

*** Nivel de significancia en $99 \%$

Fuente: elaboración propia.

Los resultados de las estimaciones que toman como variable dependiente el promedio estatal anual de IED japonesa automotriz por empresa nipona se 
muestran en la tabla 4. Al igual que en estimaciones anteriores, se obtuvo un resultado favorecedor en el criterio AIC para el modelo SAR, sin embargo con valores cercanos en el modelo SEM. Dado lo anterior se mantiene la postura señalada, donde se menciona que el modelo SEM es el que más se adecua para el análisis de aglomeración de IED.

En el modelo 7 (SAR) y en el modelo 8 (SEM) se confirma la significancia estadística para las variables de disponibilidad laboral (dislab), infraestructura en transportación (transno) y distancia geográfica (distgeo). Además, se muestra una significancia de $95 \%$ para el índice de autorregresión espacial ( $\rho$ ) y 99\% para el índice de error de autocorrelación espacial $(\lambda)$. Lo anterior reafirma la presencia de efectos espaciales positivos de la IED japonesa en la región del Bajío. Específicamente, el aumento de IED japonesa en $1 \%$ en uno de los estados analizados aumenta el $0.25 \%$ de presencia japonesa en estados vecinos.

\section{Tabla 4}

Resultados de la regresión con dependencia espacial (lnIEDEMP)

\begin{tabular}{l|c|c|c}
\hline & Modelo 7 & Modelo 8 & Modelo 9 \\
\hline Variable & SAR & SEM & SAC \\
\hline PIB per cápita (lnpibpc) & 1.7136 & 1.8749 & -1.2544 \\
\hline Costos salariales (lnsalar) & $(1.31)$ & $(1.20)$ & $(-0.25)$ \\
\hline Disponibilidad laboral (lndislab) & 7.7129 & 11.418 & 19.0157 \\
\hline & $(0.73)$ & $(0.88)$ & $(1.07)$ \\
\hline Infraestructura en transportación & $-1.8335^{* * *}$ & $-1.8814^{* * *}$ & 0.0351 \\
\hline (ntransno) & $(-5.83)$ & $(-4.67)$ & $(0.02)$ \\
\hline Distancia geográfica (lndistgeo) & $6.6696^{* * *}$ & $5.9603^{* *}$ & -5.8837 \\
\hline & & & \\
\hline Constante & $(2.64)$ & $(2.50)$ & $(-1.20)$ \\
\hline & $-7.1104^{* * *}$ & $-6.323^{* * *}$ & - \\
\hline$\rho$ & $(-3.22)$ & $(-3.04)$ & - \\
\hline$\lambda$ & 30.8271 & 10.1314 & - \\
\hline & $(0.47)$ & $(0.14)$ & - \\
\hline$\varphi$ & $0.2499^{* *}$ & & -0.3113 \\
\hline & $(2.39)$ & & $(-1.31)$ \\
\hline & & $0.2627^{* * *}$ & $0.5145^{* * *}$ \\
\hline & & $(2.73)$ & $(4.98)$ \\
\hline & & -6.4866 & \\
\hline
\end{tabular}


Cinthia Dinorah Manzano-Ramos y Leo Guzmán-Anaya

\begin{tabular}{l|c|c|c}
\hline & Modelo 7 & Modelo 8 & Modelo 9 \\
\hline Variable & SAR & SEM & SAC \\
\hline AIC & 855.4582 & 852.9634 & 842.0645 \\
\hline Delta AIC & 13.3937 & 10.8989 & 0.0000 \\
\hline Akaike weights & 0.7761 & 0.2229 & 0.0010 \\
\hline Número de observaciones & 133 & 133 & 133 \\
\hline Número de grupos & 7 & 7 & 7 \\
\hline
\end{tabular}

Notas: (1) Variable dependiente: iedemp (promedio de IED japonesa por número de empresa del sector automotriz mexicano, por entidad federativa 1999-2017). (2) Números en paréntesis indican z (o t para el caso de efectos fijos) estadístico. (3) Todos los datos se encuentran en logaritmos.

* Nivel de significancia en $90 \%$

** Nivel de Significancia en $95 \%$

*** Nivel de significancia en $99 \%$

Fuente: elaboración propia.

\section{Conclusiones}

La presente investigación se enfocó en comprobar los determinantes de aglomeración de la inversión japonesa del sector automotriz, tomando el caso de la región del Bajío. De acuerdo con los resultados obtenidos, se encontraron ciertos factores para explicar el comportamiento de la inversión en cuanto a su localización. Además, el análisis pudo observar dependencia espacial en los datos de IED japonesa al considerar variables económicas regionales.

Los coeficientes de autocorrelación espacial bajo diferentes especificaciones señalan la presencia de efectos espaciales positivos de la IED japonesa en la región del Bajío. Específicamente, aumentos de IED en un estado van de la mano con incrementos en inversión en estados vecinos. Lo anterior parece señalar que no existen efectos competitivos en la atracción de IED entre estados conocido en la literatura como "crowding out". Dicho lo anterior, es preciso mencionar que de acuerdo con los resultados obtenidos, en el análisis de regresión espacial existe interdependencia espacial en los estados pertenecientes a la zona del Bajío, donde la IED japonesa sigue un patrón de localizarse primeramente en algún estado y esto provoca atracción a estados vecinos. Esta aglomeración puede ser resultado de buscar y lograr ventajas estratégicas para las firmas japonesas permitiéndoles aprovechar economías de escala, mercados laborales especializados y grupos de proveedores. 
Además, los resultados de la investigación sugieren cuatro factores determinantes de la aglomeración de la IED de origen japonés en la región del Bajío. Específicamente, los resultados empíricos señalan las variables relacionadas con el PIB per cápita, la disponibilidad laboral, la infraestructura en transportación y la distancia geográfica. ${ }^{4}$

La variable de PIB per cápita registra una relación positiva y significativa, señalando que el tamaño del mercado ayuda a explicar la aglomeración de la IED japonesa. Los resultados confirman lo reportado por Blanc-Brude et al. (2014) y por Coughlin y Segev (2000). En este sentido, empresas japonesas parecen elegir ubicarse en lugares con características económicas similares.

Por otro lado, la variable "disponibilidad laboral" presentó significancia estadística; sin embargo, los coeficientes tienen el signo contrario esperado. Lo anterior podría reflejar la preferencia de empresas japonesas por establecerse en mercados laborales especializados y disposición por pagar salarios más altos. Además, la variable PIB per cápita puede estar afectando las estimaciones, capturando los efectos del capital humano, como es señalado por Blanc-Brude et al. (2014).

En lo que respecta a la variable "infraestructura de transporte", los resultados muestran una relación positiva entre la presencia de infraestructura y la aglomeración de la IED japonesa. Estos hallazgos van de acuerdo con lo encontrado por Smith y Florida (1994) y por Boudier-Bensebaa (2005). Para empresas japonesas la infraestructura es una variable determinante en el proceso de decisión para establecerse en alguna región.

La variable "distancia" resultó estadísticamente significativa, señalando que la IED japonesa en México se aglomera y elige su ubicación tomando en cuenta la cercanía a Estados Unidos. Estos hallazgos refuerzan los resultados empíricos previos que señalan que la IED japonesa elige su ubicación tomando en cuenta la distancia hacia su mercado meta (Blanc-Brude et al., 2014; Coughlin \& Segev, 2000; Villaverde \& Maza, 2015).

Respecto a los costos salariales, la variable no resultó estadísticamente significativa en ninguno de los modelos. Esto no necesariamente indica que los costos salariales no son considerados por los japoneses al establecerse en una

4. Se estimaron modelos adicionales que incluían aspectos del contexto social y político como proxies de políticas públicas de los estados analizados. Sin embargo, en ningún caso y bajo ninguna especificación se reportaron resultados estadísticamente significativos, por lo que no son incluidos en el presente análisis. Los resultados están disponibles a petición de consulta. 
entidad, sino que las estimaciones pudieron demostrar un mayor peso a las variables económicas y regionales que sí presentaron resultados significativos. Además, variables como el "PIB per cápita" o "disponibilidad laboral" pueden estar absorbiendo el efecto relacionado con los costos salariales.

Los resultados reportados muestran que inversionistas japoneses optan por aglomerarse en entidades con otras empresas japonesas de la misma industria. Este tipo de comportamiento relaciona a la IED japonesa con la preferencia por colaborar y estar en cercanía con empresas de su misma nacionalidad, con las que comparten intereses y aprovechan economías de escala, desarrollo de proveedores y competencia directa. Esto reduce costos de transacción y genera ahorros de tiempo y costos en investigación respecto a la localización en el país receptor. En este sentido se tienen antecedentes de firmas rivales japonesas compartiendo centros de investigación y desarrollo, líneas de producción y entrenamiento de proveedores.

Finalmente, se sugiere para estudios futuros explorar el análisis espacial con unidades geográficas más desagregadas; por ejemplo, con datos municipales. Además, se podría complementar el análisis tomando estudios de caso con técnicas cualitativas para tener un entendimiento más completo de la aglomeración japonesa en México.

\section{Referencias}

Alfaro, L., \& Chen, M. (2014). The Global Agglomeration of Multinational Firms. Journal of International Economics, 94(2), 263-276. doi: https:// doi.org/10.1016/j.jinteco.2014.09.001

Belderbos, R., \& Carree, M. (2002). The Location of Japanese Investments in China: Agglomeration Effects, Keiretsu, and Firm Heterogeneity. Journal of the Japanese and International Economies, 16(2), 194-211. doi: https:// doi.org/10.1006/jjie.2001.0491

Belotti, F., Hughes, G., \& Mortari, A. P. (2017). Spatial Panel-data Models Using Stata. Stata Journal, 17(1), 139-180. doi: https://doi. org/10.1177/1536867X1701700109

Blanc-Brude, F., Cookson, G., Piesse, J., \& Strange, R. (2014). The FDI location decision: Distance and the effects of spatial dependence. International Business Review, 23(4), 797-810. doi: https://doi.org/10.1016/j.ibusrev.2013.12.002 
Blonigen, B. A., Davies, R. B., Waddell, G. R., \& Naughton, H. T. (2004). FDI in Space: Spatial Autoregressive Relationships in Foreign Direct Investment (National Bureau of Economic Research NBER Working Paper 10939). doi: https://doi.org/10.3386/w10939

Bobonis, G. J., \& Shatz, H. J. (2007). Agglomeration, Adjustment, and State Policies in the Location of Foreign Direct Investment in the United States. The Review of Economics and Statistics, 89(1), 30-43. doi: https://doi. org/10.1162/rest.89.1.30

Boudier-Bensebaa, F. (2005). Agglomeration economies and location choice: Foreign direct investment in Hungary. Economics of Transition, 13(4), 605-628. doi: https://doi.org/10.1111/j.0967-0750.2005.00234.x

Buchanan, B., \& Le, Q., \& Rishi, M. (2012). Foreign direct investment and institutional quality: Some empirical evidence. International Review of Financial Analysis, 21, 81-89. https://doi.org/10.1016/j.irfa.2011.10.001 Canales, G. (2010). La Inversión Extranjera Directa en México; Políticas Públicas para el Desarrollo (Proyecto de Investigación Aplicada. Maestría en Administración Pública y Política Pública. Instituto Tecnológico y de Estudios Superiores de Monterrey. Campus Ciudad de México). Recuperado de https:// repositorio.tec.mx/bitstream/handle/11285/629262/33068001086794. pdf? sequence $=1$

Cheng, L. K., \& Kwan, Y. K. (2000). What are the determinants of the location of foreign direct investment? The Chinese experience. Journal of International Economics, 51(2), 379-400. doi: https://doi.org/10.1016/ S0022-1996(99)00032-X

Coughlin, C. C., \& Segev, E. (2000). Foreign Direct Investment in China: A Spatial Econometric Study. The World Economy, 23(1), 1-23. doi: https:// doi.org/10.1111/1467-9701.t01-1-00260

Du, J., Lu, Y., \& Tao, Z. (2008). FDI Location choice: Agglomeration vs Institutions. International Journal of Finance \& Economics, 13(1), 92-107. doi: https://doi.org/10.1002/ijfe.348

Friedman, J., Gerlowski, D. A., \& Silberman, J. (1992). What Attracts Foreign Multinational Corporations? Evidence from Branch Plant Location in the United States. Journal of Regional Science, 32(4), 403-418. doi: https:// doi.org/10.1111/j.1467-9787.1992.tb00197.x

Fukao, K., Kravtsova, V., \& Nakajima, K. (2014). How important is geographical agglomeration to factory efficiency in Japan's manufacturing sector? Annals 
of Regional Science. 52, 659-696. doi: https://doi.org/10.1007/s00168014-0601-9

Guimarães, P., Figueiredo, O., \& Woodward, D. (2000). Agglomeration and the Location of Foreign Direct Investment in Portugal. Journal of Urban Economics, 47(1), 115-135. doi: https://doi.org/10.1006/juec.1999.2138 $\mathrm{He}, \mathrm{C}$. (2003). Location of foreign manufacturers in China: Agglomeration economies and country of origin effects. Papers in Regional Science, 82(3), 351-372. doi: https://doi.org/10.1007/s10110-003-0168-9

Head, C. K., Ries, J. C., \& Swenson, D. L. (1999). Attracting foreign manufacturing: Investment promotion and agglomeration. Regional Science and Urban Economics, 29(2), 197-218. doi: https://doi.org/10.1016/ S0166-0462(98)00029-5

Head, K., Ries, J., \& Swenson, D. (1995). Agglomeration benefits and location choice: Evidence from Japanese manufacturing investments in the United States. Journal of International Economics, 38(3-4), 223-247. doi: https:// doi.org/10.1016/0022-1996(94)01351-R

Horn, S. A., \& Cross, A. R. (2016). Japanese production networks in India: spatial distribution, agglomeration and industry effects. Asia Pacific Business Review, 22(4), 612-640. doi: https://doi.org/10.1080/136023 81.2016.1168625

Jordaan, J. (2009). Foreign Direct Investment, Agglomeration and Externalities. Empirical Evidence from Mexican Manufacturing industries. London: Routledge. doi: https://doi.org/10.4324/9781315582788

Kang, Y., \& Jiang, F. (2012). FDI location choice of Chinese multinationals in East and Southeast Asia: Traditional economic factors and institutional perspective. Journal of World Business, 47(1), 45-53. doi: https://doi. org/10.1016/j.jwb.2010.10.019

Kotabe, M. (1993). The promotional roles of the state government and Japanese manufacturing direct investment in the United States. Journal of Business Research, 27(2), 131-146. doi: https://doi.org/10.1016/01482963(93)90019-L

Krugman, P. (1991). Increasing Returns and Economic Geography. The Journal of Political Economy, 99(3), 483-499.

Lugo-Sánchez, M.G. (2018). The Role of Public Policies in Attracting Japanese FDI in Mexico. En M. Falck Reyes y L. Guzmán-Anaya (Coords.), Japanese Direct Investment in Mexico's Transport Equipment Sector. Macro Impact 
and Local Responses (pp. 91-101). Singapur: Springer. doi: https://doi. org/10.1007/978-981-10-7718-0

Lien, Y. C., \& Filatotchev, I. (2015). Ownership characteristics as determinants of FDI location decisions in emerging economies. Journal of World Business, 50(4), 637-650. doi: https://doi.org/10.1016/j.jwb.2014.09.002

Lincoln, J. R., \& Shimotani, M. (2010). Business Networks in Postwar Japan: Whither the Keiretsu? In A. M. Colpan, T. Hikino, \& J. R. Lincoln (Eds.), The Oxford Handbook of Business groups (pp. 127-156). New York: Oxford University.

Marshall, A. (1961). Principles of Economics ( ${ }^{\text {th }}$ edition). New York: Palgrave Macmillan.

Ortega, P. (2015). Política nacional e inversión extranjera directa en México en el entorno internacional. Revista Iberoamericana de Contaduría, Economía y Administración, 4(8), 33-55. Recuperado de http://www.ricea.org.mx/ index.php/ricea/article/view/27/63

Porter, M. (1986). Changing Patterns of International Competition. California Management Review, 28(1), 9-40. doi: https://doi.org/10.2307/41165182

ProMéxico. (2016). La industria Automotriz Mexicana: Situación actual, Retos y Oportunidades. Recuperado de https://www.gob.mx/promexico

ProMéxico. (2017). Diez años del Acuerdo de Asociación Económica México Japón. México: ProMéxico.

Secretaría de Economía. (2017). Inversión Extranjera Directa en México y en el Mundo. Recuperado de https://www.gob.mx/se/articulos/que-es-lainversion-extranjera-directa

Smith, D. F., \& Florida, R. (1994). Agglomeration and Industrial Location: An Econometric Analysis of Japanese-Affiliated Manufacturing Establishments in Automotive-Related Industries. Journal of Urban Economics, 36(1), 23-41. doi: https://doi.org/10.1006/juec.1994.1024

Tinoco-García, M.J., \& Guzmán-Anaya, L. (2020). Factores Regionales de Atracción de Inversión Extranjera Directa en México. Análisis Económico, 35(88), 89-117.

Tokunaga, S., \& Jin, S. (2007). An Empirical Analysis of Agglomeration Effects on the Location Choice of Japanese Electronics Firms in China Using Provincial Data. Studies in Regional Science, 37(1), 175-185. doi: https:// doi.org/10.2457/srs.37.175 
Villaverde, J., \& Maza, A. (2015). The determinants of inward foreign direct investment: Evidence from the European regions. International Business Review, 24(2), 209-223. doi: https://doi.org/10.1016/j.ibusrev.2014.07.008

Weber, A., \& Friedrich, C. J. (1929). Theory of the Location of Industries. Chicago: The University of Chicago.

Zhou, C., Delios, A., \& Yang, J. Y. (2002). Locational Determinants of Japanese Foreign Direct Investment in China. Asia Pacific Journal of Management, 19(1), 63-86. doi: https://doi.org/10.1023/A:1014839607180 